\title{
LOS LUGARES DE MEMORIA COMO PATRIMONIO: PROPUESTA DE ITINERARIOS EN CARTAGENA DURANTE LA II REPÚBLICA (1931-1936)
}

\author{
THE PLACES OF MEMORY AS HERITAGE: \\ PROPOSED ITINERARIES IN CARTAGENA \\ DURING THE II REPUBLIC (1931-1936)
}

\author{
Carmen Egea Ibarra
}

\section{RESUMEN}

Este trabajo tiene como fin elaborar una guía de itinerarios históricos de la ciudad de Cartagena durante los años de la II República. Se pretende preservar un patrimonio inmaterial, aquellos lugares de la memoria de la ciudad republicana que hacen referencia a los símbolos de identidad de una colectividad. A sus monumentos silenciosos y silenciados. El objetivo es recuperar el contexto histórico de los edificios y calles y destacar su importancia didáctica, memorialista y cultural, teniendo en cuenta la Ley de Memoria Histórica.

El estudio se organiza en tres capítulos: el primero aborda el contexto histórico, Cartagena en los años 30; el segundo titulado Ciudad y Memoria, donde se analiza la estructura de la ciudad siguiendo a Kevin Lynch. El último capítulo desarrolla tres rutas: la política, destacando el Arsenal y el Ayuntamiento; la cultural con el Ateneo y la Universidad Popular y por último la ruta carcelaria con el viejo Penal, el Castillo de San Julián y la Cárcel de San Antón.

Palabras claves: Cartagena, II República, patrimonio, memoria histórica, itinerarios urbanos.

\section{ABSTRACT}

This work aims to develop a guide of historical itineraries of the city from Cartagena during the years of the Second Republic. The aim is to preserve an intangible heritage, those places in the memory of the republican city that refer to the identity symbols of a community. To its silent and silenced monuments. The objective is to recover the historical context of the buildings and streets and to emphasise its didactic, memorialist and cultural importance, taking into account the Law of Historical Memory.

This research is divided in three chapters: the first deals with the historical context, Cartagena in the 1930s; the second titled City and Memory, where the structure of the city is analysed following Kevin Lynch. The last chapter develops three routes: politics, highlighting the Arsenal and the City Council; the cultural with the Ateneo and the Popular University and finally the prison route with the old Penal, the Castle of San Julián and the Prison of San Antón. Keywords: Cartagena, II Republic, heritage, historical memory, urban itineraries. 


\section{INTRODUCCIÓN}

Cartagena fue declarada Conjunto Histórico por Real Decreto de 1980, refrendado con la categoría de Bien de Interés Cultural con la Ley Nacional de Patrimonio de 1985. A pesar de ello se ha asistido a la destrucción de sus bienes protegidos a tenor de ciertos intereses urbanísticos.

La Ley 52/2007, de 26 de diciembre, conocida como Ley de Memoria Histórica, “... sienta las bases para que los poderes públicos lleven a cabo políticas públicas dirigidas al conocimiento de nuestra historia y al fomento de la memoria democrática". Añade que los ciudadanos tienen derecho "... a que los símbolos públicos sean ocasión de encuentro y no de enfrentamiento, ofensa o agravio". Confluye en el deseo de "... fomentar los valores constitucionales y promover el conocimiento y la reflexión sobre nuestro pasado, para evitar que se repitan situaciones de intolerancia y violación de derechos humanos como las entonces vividas"

El objetivo de este trabajo es realizar una aproximación a la realidad histórica y patrimonial de la ciudad de Cartagena durante los años de la II República, que contextualice y permita la elaboración de una guía de itinerarios históricos de la ciudad levantina. El viaje a una memoria desconocida por marginada, cuando no deformada y ocultada de forma deliberada durante la dictadura militar del general Franco, cuya sombra se proyecta sobre el presente en el tema que nos ocupa. La Ley de Memoria Histórica se incumple de manera sistemática por los ayuntamientos, además de carecer de consignación en los Presupuestos Generales del Estado, lo que remarca la trascendencia ideológica que conlleva la apropiación del pasado.

El interés es la preservación de lo que se considera un patrimonio inmaterial: los lugares de la memoria de la ciudad republicana, aquellos que hacen referencia a los símbolos de identidad de una colectividad. La propuesta presentada trata de ofrecer un recorrido por las huellas de un tiempo que configuran nuestro ser social, nuestro ser histórico, un recorrido a los vestigios de la República en Cartagena.

El enfoque propuesto pretende recuperar el contexto histórico que rodea a cada lugar elegido junto con la inmaterialidad de su valor patrimonial al objeto de subrayar su significación didáctica, memorialista y cultural. Los criterios de selección apuntan justamente a la doble consideración histórica y patrimonial. Se desea poner en valor lugares emblemáticos cargados de referencias históricas, de significantes de memoria.

El trabajo se estructura en tres capítulos. El primero contempla el contexto histórico abordado -Cartagena en los años 30-, necesario para poder insertar los lugares de memoria analizados. Se singulariza la ciudad y su término dentro del conjunto provincial. Se subraya su carácter industrial y militar, junto con la presencia de una clase obrera organizada. Se destacan sus problemas de habitabilidad, sanitarios e higiénicos. Se perfilan sus coordenadas de la vida cotidiana, la cuestión educativa, el arraigo de la ideología republicana y los grandes planteamientos del reformismo republicano. Lynch, K. (2015). La imagen de la ciudad. Barcelona: Gustavo Gili.

A los mimbres económicos, sociales y políticos sigue la huella republicana, analizada en el apartado de Ciudad y memoria. Siguiendo a Kevin Lynch analizamos la estructura de la ciudad en sendas, bordes, barrios, nodos e hitos. Prestamos atención al deseo de implantar una nueva memoria en sustitución de la anterior, centrándonos en la carga simbólica del nomenclátor callejero. La ciudad experimentó una profunda remodelación, empezando por la destrucción de los símbolos del pasado y la rotulación de las calles, donde se borraron denominaciones que miraban al pasado menos aceptable para los moldes democráticos que se abrían paso, dejando sitio a designaciones de alcance social y político acordes con los nuevos tiempos.

Concluimos con la propuesta de una Guía de itinerarios históricos, donde planteamos tres rutas. La ruta política, con las calles como soporte del escenario sugerido, con indicaciones a los hitos más destacados, empezando por el Arsenal, desde cuya dársena Alfonso XIII tomó el camino del exilio. De no menor relieve es el Ayuntamiento, desde cuyo balcón se proclamó la II República y los locales de las organizaciones políticas y sindicales que dinamizaron toda

1 Boletín Oficial del Estado, 27 de diciembre de 2007. 
la etapa con sus reivindicaciones sociales. La segunda ruta es la cultural, privilegiando la sustitución de la enseñanza religiosa, la creación de grupos escolares y el papel del Ateneo y de la Universidad Popular. Cerramos la Guía con la ruta carcelaria. Lugares de represión, especialmente utilizados durante el segundo bienio republicano: el viejo Penal, el Castillo de San Julián y la Cárcel de San Antón, levantada ahora para dignificar las condiciones materiales de los reclusos.

\section{CARTAGENA EN LOS AÑOS 30}

En 1930 Cartagena albergaba una población de 102.518 habitantes, 52.918 hombres y 49.600 mujeres. El núcleo urbano contaba con 36.024 habitantes, en tanto los barrios extramuros sumaban 18.823 y las diputaciones rurales 47.671. (Egea, 1996: 307) Era la segunda urbe de la provincia, inmediatamente detrás de la capital.

El lugar se singularizaba como núcleo industrial: minería, metalurgia, construcción naval, vidrio, fundiciones de pequeño porte y sector químico. De relieve eran también las ocupaciones conectadas al tráfico portuario y a la construcción. Todo ello daba pie a la formación de una nutrida y bien organizada clase obrera, en tanto la sociedad en general se deslizaba hacia planteamientos progresistas, con una clara traducción electoral. El contrapunto venía dado por el importante peso de la población castrense al albergar una sólida plaza militar y una base naval de primera magnitud, lo que contribuía a explicar el desnivel entre hombres y mujeres.

Las grandes factorías se levantaban en los extramuros de la ciudad: Sociedad Española de Construcción Naval y Arsenal (Barrio de La Concepción), Fundición de Plomo de Peñarroya y Fábrica del Vidrio (Barrio de Santa Lucía) y Productos Químicos en el Hondón. Se agregaba un interminable cómputo de talleres artesanales que tomaban asiento en casi todas las vías de la ciudad: licores, bebidas gaseosas, jabones, sacos, envases de madera, trabajo de metales, de somier y camas de hierro, molduras, lunas de espejo, guano, sombrererías, cera, aserrerías, pastas para sopa, chocolaterías o muebles, que daban empleo a una legión de operarios (Casal, 1933).

La ciudad se había desarrollado marcada por la impronta militar y la accidentada topografía del asentamiento, conformada por una estrecha franja litoral y las cinco colinas que se alzaban en su recinto: Concepción, Despeñaperros, San José, Monte Sacro y Molinete. De ello se derivaba la existencia de dos ciudades: la burguesa y la popular. En la parte llana las calles principales con las mejores casas, donde habitaban las personas más acomodadas. En las alturas las clases con menos recursos, barriadas donde se veía con toda crudeza las condiciones más adversas, el confuso hacinamiento de los desfavorecidos. Lo indicado ha conformado sus espacios urbanos (Andrés, 1981; Egea, 1999; Celdrán, 2008 y Ros. 2010).

El analfabetismo alcanzaba al $43 \%$ de la población; 36\% hombres y $50 \%$ mujeres (Egea, 2006: 39). Se contaba con tres Graduadas de Niños -San Fulgencio, San Leandro y San Isidoro-, que habían sido las primeras de España. Las niñas disponían de tres: Santa Florentina, $\mathrm{N}^{\mathrm{a}}$. $S^{\mathrm{a}}$. del Carmen y $\mathrm{N}^{\mathrm{a}}$. $\mathrm{S}^{\mathrm{a}}$. de la Caridad. Se añadían nueve escuelas unitarias y seis de párvulos. Existía un Instituto de Segundo Enseñanza, en la Plaza del Parque; el Conservatorio Municipal de Música y Declamación se encontraba en la Calle de Pi y Margall; la Escuela Superior y Elemental de Trabajo en la Alameda de San Antón-; La Escuela Profesional de Comercio en Cuatro Santos y la Escuela de Capataces y Facultativos de Minas y Fábricas Metalúrgicas, estaba instalada en la Sociedad Económica de Amigos del País (Casal, 1933: 91-93).

Los partidarios de la República se fueron incrementando en proporción directa al creciente deterioro de la Monarquía, asentada en la corrupción política, multiplicándose a partir de su identificación con la dictadura de Miguel Primo de Rivera (1923-1930). El postrer impulso vino de la mano de la sublevación republicana de Jaca de 12 de diciembre de 1930 y, sobre todo, de su dramático desenlace, el fusilamiento el día 14 de los considerados responsables, los capitanes Galán y García Hernández. Como protesta se convocó una huelga general para los días 15 y 16, firmemente secundada en Cartagena. El gobierno respondió con la declaración del estado de guerra y la detención de los instigadores del plante (Martínez Leal, 1993: 79-80). 
La represión terminó de aislar a la Corona. La República nace por dos razones: debido a revolución del pueblo y debido a la mala dirección de la Restauración. (Vinyals i Garrido, 2016). Con este ambiente de represión se inició 1931. El 12 de de abril se convocaron elecciones municipales. La derecha acudió por separado. La izquierda formó la Conjunción Republicano-Socialista. Los resultados fueron, como los del resto del país, un plebiscito contra los Borbones: 7.004 votos de la Conjunción por 3.942 monárquicos, lo que se tradujo en 28 concejalías frente a 17 de éstos. Con aquel respaldo, Cartagena proclamó la República el 14 de abril (Martínez Leal, 1993: 88 y Franco, 2002: 49-50).

Tres etapas pueden distinguirse en la evolución política de la República: el Bienio socialazañista (1931-1933), el Bienio radical-cedista (1933-1936) y el Frente Popular (febrero-julio de 1936). La República fue un proyecto reformista, que se concretó durante el primer bienio, conocido como bienio social-azañista, un gobierno de republicanos de izquierda y socialistas. Este gabinete, dirigido por Manuel Azaña, adoptó un sistema reformista por medio de una labor legislativa, que quiso enfrentarse a los graves problemas heredados de la Monarquía: la Iglesia, el Ejército y la estructura agraria semifeudal, sin olvidar la cuestión social y regional.

\section{CIUDAD Y MEMORIA}

En los últimos años la memoria colectiva figura como un nuevo campo historiográfico. Ha sido señalada como "la materia misma de la historia" (Cuesta, 1998: 203). Dentro de la disciplina, Pierre Nora (2008: 19) aborda los lugares de la memoria, que aplica a "toda unidad significativa, de orden material o ideal, de la cual la voluntad de los hombres o el trabajo del tiempo ha hecho un elemento simbólico del patrimonio memorial de cualquier comunidad".

La importancia de recordar es un factor esencial en el uso del patrimonio, pero además, puede deducirse del mismo un sentido moral al considerar que la generación presente tiene una deuda pendiente con el pasado. El proyecto llamado Patrimonio Aplicado, resolución de conflictos a través de la historia cultural, centrado en el Museo del Condado de Kalmar, su objetivo es estudiar de qué manera y para qué resultados el patrimonio cultural se puede aplicar para curar heridas en una sociedad dividida. (Wollentz, 2014).

Las ciudades aparecen como lugares de memoria y dentro de ellas las calles ocupan un lugar relevante. Las calles reflejan el devenir ciudadano. La historia de una ciudad "... se condensa y se conmemora en sus calles (Sánchez Costa, 2009). Forman parte de la cultura política (Cabrera, 2010). Están cargadas de simbolismo político bajo la influencia de las diferentes ideologías dominantes en cada periodo histórico (Henales, 2017).

Kevin Lynch (2015) ha analizado la estructura de las ciudades mediante cinco elementos: sendas, bordes, barrios, nodos e hitos, definidos por sus connotaciones sociales. El primero se refiere a las calles más transitadas, circunscritas en nuestro caso al casco antiguo, donde se destacan dos ejes principales, como se verá más adelante. El más importante está formado por la Calle Mayor, la Plaza de San Sebastián, las Puertas de Murcia y la Calle del Carmen. Son las vías consideradas de primer orden en la clasificación municipal de entonces (Casal, 1933: 19). El segundo eje está integrado por las de segundo orden, como son las de Cuatro Santos, Duque y San Diego. Hay que añadir las vías próximas de San Miguel, Osuna, Campos, Aire y Jara.

Los bordes suponen la ruptura de la continuidad urbana. Son los aledaños y los altozanos de las colinas que se alzan en el núcleo: Concepción, Despeñaperros, San José, Monte Sacro y Molinete, este último como punto central del meretricio cartagenero, donde no faltan los escándalos y las pendencias. Lugares cruzados por vías de tercer y cuarto orden: Plaza y Calle de la Aurora, Cuesta del Maestro Francés, Caramel, Ignacio García, Lizana, Morería Baja, Montanaro, Martín Delgado, Marango, Paraíso, Seña, Sambazart, San Vicente o las de San Cristóbal la Larga y la Corta (Casal, 1986). Su estado solía ser descuidado, como ocurría en la de la Gloria: “... se encuentra en un estado tal, que hasta las pobres caballerías les cuesta trabajo transitar por aquel sitio"2. En ellas se emplazaban las viviendas obreras y sus centros de reunión.

Los barrios, extramuros a la ciudad, estaban integrados por los de La Concepción, Peral, San Antón, Santa Lucía y Los Dolores, donde se mantenían las diferencias económicas.

2 Cartagena Nueva, 11 de noviembre de 1931. 
Alejados de los centros de decisión, su protagonismo político era irrelevante. En los barrios obreros, con las excepciones de rigor, las calles eran de cuarto y quinto orden.

Los nodos se insertan en las vías principales, donde se centraba la actividad mercantil y social de los grupos privilegiados. Escenarios de desfiles y actos públicos: la Calle Mayor, la Plaza de San Sebastián, las Puertas de Murcia y Calle del Carmen, con la Plaza del Ayuntamiento como obligada referencia. Allí toman asiento las mansiones de la burguesía, las mejores cafeterías y establecimientos comerciales. Allí alternan los centros de sociabilidad y los círculos políticos de aquellas clases (Pérez Rojas, 1986: 103-110).

Los hitos se destacan por sus significados, por la carga simbólica que ostentan, edificios y lugares, que varían en cada época histórica. Trataremos de recuperar el sentido que tuvieron los más destacados durante los años de la II República: Ayuntamiento, Arsenal, Ateneo, Universidad Popular, Penal...

Durante la II República, las grandes ideas se acompañan de alusiones cotidianas. Los cambios en el callejero, buscaban sustituir menciones religiosas, militares y monárquicas por denominaciones adecuadas a los nuevos tiempos, en recuerdo de personalidades y de categorías abstractas. De este modo, se recuperará la memoria del Cantón y del republicanismo decimonónico; se añadirá la más reciente, con los mártires de la República; la del presente inmediato, con obligadas menciones a la República y al 14 de Abril- y se contemplarán los nombres de los republicanos y socialistas fallecidos a partir de entonces. No hay ninguna calle asociada al nombre de una mujer, lo que si ocurrió durante la Guerra Civil. (Victoria, 1993).

Las permutas en el nomenclátor traducirán la correlación de las fuerzas políticas en las distintas corporaciones municipales. En Cartagena fueron republicanos de diversos matices y socialistas los que llevaron a cabo las mociones oportunas, aceptando los segundos vías de menor importancia. La rememoración fue por tanto republicana y socialista. Sólo hubo una calle de impronta anarquista, que llegó de la mano del concejal Antonio Ros, del Partido Radical Socialista, una izquierda republicana. La calle del General Aznar -antes de Jara- pasó a denominarse de Salvador Seguí (Noi del Sucre) ${ }^{3}$.

Los canjes apenas se demoraron, concretándose con una sola excepción al primer bienio republicano, el reformista. El 17 de abril de 1931 se constituyó el nuevo Ayuntamiento. Por unanimidad fue elegido alcalde Francisco Pérez Lurbe, del Partido Radical, la opción más templada del republicanismo. En la segunda sesión, celebrada el 24 de abril, el concejal Ramón Navarro Vives -de la misma formación - propuso las siguientes alteraciones.

TABLA 1: renombramiento del callejero

NOMBRE ANTIGUO

Avenida del 13 de Septiembre

\begin{tabular}{ll}
\hline Paseo de Alfonso XIII & Paseo de La Libertad \\
\hline Plaza del Rey & Plaza de la República \\
\hline Plaza de García Alix & Plaza de García Hernández \\
\hline Parque de la Concepción (Torres) & Parque de los Cantonales \\
\hline Calle de la Reina María Cristina & Calle del Doctor Cárceles \\
\hline Paseo de Alfonso XII & Paseo Desconocidos Mártires de la Libertad \\
\hline Plaza de Jaime Bosch & Plaza de Antonete Gálvez \\
\hline Avenida del General Muñoz Cobos & Paseo del Capitán Galán \\
\hline Calle Real & Paseo de Pablo Iglesias \\
\hline
\end{tabular}

Fuente: $E l$ Eco de Cartagena, 25 de abril de 1931.

\section{NOMBRE PROPUESTO}

Avenida del 14 de Abril

3 El Eco de Cartagena, 20 de junio de 1931 
Se buscó la mayor resonancia posible. Salvo la de María Cristina, situada en el Ensanche, las demás vías eran de primer orden, o bien constituían los ejes principales de la ciudad o eran muy céntricas. Los relevos de reyes, reinas y demás indicaciones monárquicas como el ministro García Alíx o el alcalde Jaime Bosch parecían obligados. En su lugar aparecían alusiones a la Libertad, La República, Galán, García Hernández, Mártires de la Libertad y al adalid histórico del socialismo español: Pablo Iglesias. La Avenida del 13 de Septiembre (Paseo de la Estación) era una evocación del golpe militar de Primo de Rivera-, reemplazada por la del 14 de Abril, la fecha germinal del nuevo régimen.

El movimiento cantonal mereció reiteradas distinciones. Desde el Parque de los Cantonales a los dirigentes del aquel movimiento: Antonete Gálvez y Manuel Cárceles. El Dr. Cárceles mereció una calle en el Ensanche. Muy anciano ya remitió una carta de agradecimiento al Concejo municipal ${ }^{4}$. El 16 de junio de 1931 fue recibido en loor de multitudes en la ciudad que lo vio nacer. Por entonces era jefe del Partido Republicano Federal. Acudieron a recibirlo las autoridades locales y la población en masa ${ }^{5}$.

Enlasesión del 4 de mayo de 1931 se aprobóla variación de otra tanda de calles, rectificándose algunas de las ya aprobadas. Se siguió insistiendo en la recuperación de la memoria republicana. Casimiro Bonmatí dio lectura a una moción para que se diese el nombre de Bartual a la Plaza de Los Tres Reyes. Manuel Bartual Verdejo era un líder republicano ajusticiado el 3 de marzo de 1886, tras el fracaso del movimiento que había previsto la toma del Castillo de San Julián. A iniciativa de Bonmatí se promovió el acuerdo municipal de dar su nombre a la Plaza de Los Tres Reyes 6 $\mathrm{El} \mathrm{mismo} \mathrm{ponente} \mathrm{se} \mathrm{encargaba} \mathrm{de} \mathrm{rebajar} \mathrm{la} \mathrm{carga} \mathrm{antirreligiosa} \mathrm{de} \mathrm{la} \mathrm{ponencia,} \mathrm{subrayando} \mathrm{la}$ espiritualidad del homenajeado al señalar que era "un republicano que creía en Dios", remarcando su imagen de lealtad y calificándolo de mártir?. A propuesta de Amancio Muñoz de Zafra - de la minoría socialista- se decidió que el Paseo del Muelle -de Alfonso XII- pasase a llamarse de Pablo Iglesias. Finalmente, el concejal Ramón Navarro Vives, del Partido Radical, tomó la palabra para proponer que a la calle Real se le pusiera la de la Maestranza, en lugar de Avenida de los Aliados ${ }^{8}$.

El 1 de abril de 1932 se planteó una nueva rotulación de calles. En esta ocasión presidía la sesión el radical socialista Isidro Pérez San José, nombrado alcalde en aquella misma ocasión. Uno de sus correligionarios, el concejal Marcial Morales, propuso sustituir los nombres de San Miguel y Plaza de San Ginés por los de Alcalá Zamora y Marcelino Domingo ${ }^{9}$. Dos vías céntricas, dedicadas al santoral católico, sirvieron para rendir homenaje al Presidente de la República y al ministro de Instrucción Pública, este último de la misma filiación política que el firmante de la petición.

La prensa católica se les echó encima ${ }^{10}$. A la campaña se sumó la prensa más derechista, que comparó esa labor con la escasa atención prestada a temas considerados más relevantes como el precio de las subsistencias ${ }^{11}$.

La avalancha de cambios y la reiteración de las mismas denominaciones en barrios y diputaciones despertaron el recelo de algunos concejales, como la del republicano socialista Severino Bonmatí, que lo expresó con claridad: “... hace observar la confusión que para la correspondencia supone la redundancia de unos mismos nombres dentro del término municipal de Cartagena"12. En el mismo sentido se plantearon algunas propuestas de rectificación. Ocurrió en la sesión del 8 de abril de $1932^{13}$, aprovechada por el socialista Jesús López Lorente para conseguir que la calle de la Gloria se denominase de Tomás Meabe, el que fuera presidente de las Juventudes Socialistas ${ }^{14}$. Una calle de tercer orden.

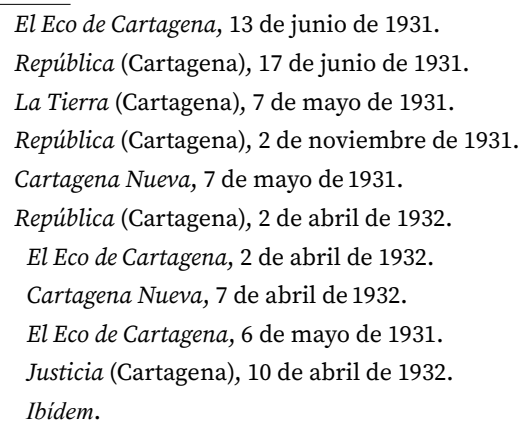


El homenaje a los fallecidos también se trasladó al callejero. Una forma de consolidar la memoria de las personas consideradas relevantes por sus méritos sociales, pero también por su identificación con los ideales republicanos. En la sesión del 5 de junio de 1931 Marcial Morales solicitó que se pusiera el nombre “... del que en vida fue entusiasta republicano Juan Francisco Mega a la actual calle de San Diego, acordándolo así el Ayuntamiento"15. Se le dedicaba uno de los ejes de la ciudad, el que se abría a la estación ferroviaria. El Dr. Mega, fallecido el 28 de diciembre de 1923, se había significado por su filantropía entre las clases populares de Cartagena ${ }^{16}$.

El 14 de julio de 1932 murió un maestro entrañable y un reconocido pedagogo: Enrique Martínez Muñoz. A él se debía la creación de las primeras escuelas graduadas de España, las de Cartagena. Era director de la Escuela Nacional Graduada San Isidoro, de la Sociedad Económica de Amigos del País y Hermano del Santo Hospital de Caridad ${ }^{17}$. Muy querido en la ciudad, la prensa solicitó del comercio el cierre de los establecimientos al paso de su entierro. El desfile funerario constituyó "una imponente manifestación de duelo. / En la presidencia figuraba el alcalde señor Pérez San José, dando guardia a la carroza fúnebre los maceros municipales"18.

Su eco oficial fue inmediato. A los dos días de su óbito, en la sesión municipal del día 16 de julio, el alcalde pidió su reconocimiento ${ }^{19}$. En la sesión del 2 de septiembre de 1932 el arquitecto municipal designó la calle número 15 del Ensanche para que llevase el nombre de Enrique Martínez Muñoz ${ }^{20}$.

El 12 de agosto de 1932 a propuesta de los socialistas Jesús López Lorente y Miguel Céspedes Pérez se acordó cambiar los nombres de las calles de San Fernando y Beatas por los de Sanchís Banús y Sánchez del Val, respectivamente. Dos vías céntricas del casco antiguo, aunque no de primer orden. La prensa de derechas añadía la oportuna coletilla: “¡Oh, la fobia laicizante!”21. El doctor José Sanchis Banús había fallecido el 22 de julio de 1932. Era catedrático de la Universidad Central de Madrid y diputado por el Partido Socialista Obrero Español, presidente del Colegio Oficial de Médicos de Madrid y destacado neuropsiquiatra. El 13 de agosto de 1931 había fallecido el también socialista Ángel Sánchez del Val, doctor en Medina y Cirugía, director del Dispensario de la Cruz Roja y teniente de alcalde del Ayuntamiento de Cartagena.

El 21 de mayo de 1933 murió Ricardo Burguete, comandante de Aviación y Jefe del Aeródromo militar de Los Alcázares, considerado un fervoroso republicano ${ }^{22}$. El 27 de mayo, bajo la presidencia del alcalde radical socialista Julio Casciaro Parodi, el concejal Marcial Morales solicitó que el nombre de Ricardo Burguete se le diese a la calle de Comedias -una vía del centro urbano, pero de segundo orden- y que constase en acta el sentimiento de la Corporación por su muerte y que se le comunicase a sus familiares ${ }^{23}$.

El 1 de noviembre de 1933 falleció Ginés de Arlés García, político, periodista y poeta, dirigente del Partido Republicano Radical Socialista, miembro de la logia masónica Tolstoi, jefe interventor de la Administración Principal de Correos, vicepresidente de la Junta Local de Protección de Menores -emplazada en la Casa del Niño- y vicepresidente de la Universidad Popular $^{24}$. La noticia conmocionó a la ciudad. Hasta el equipo de fútbol Los Diablos Rojos vistió de luto en su encuentro del domingo ${ }^{25}$.El diario República le dedicó una extensa necrológica ${ }^{26}$.

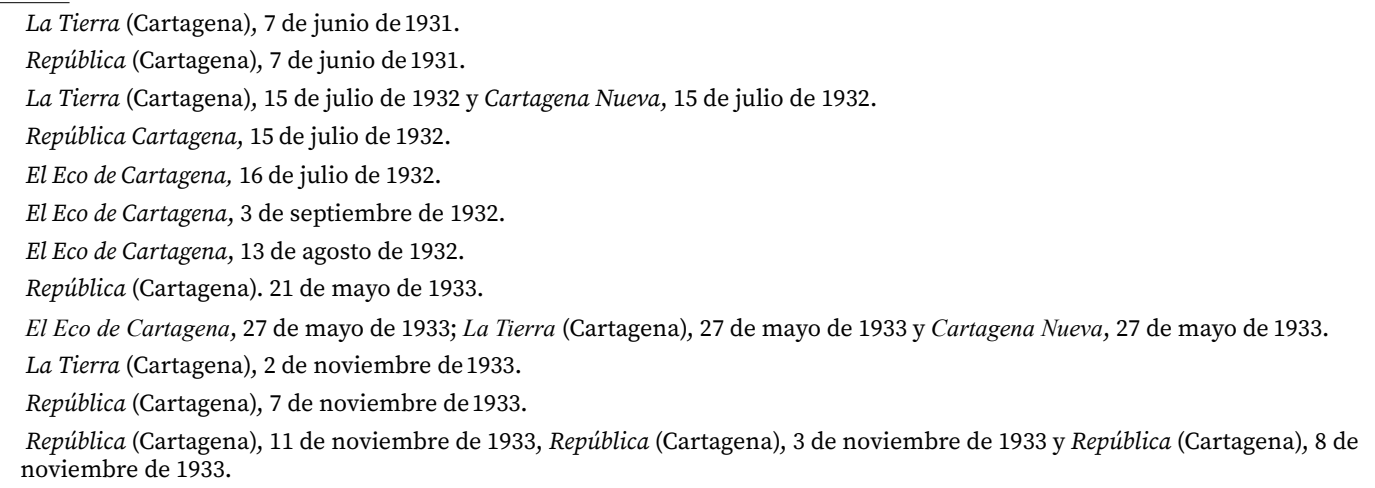


En la sesión municipal inmediata, la del 4 de noviembre, celebrada bajo la presidencia del radical Pedro Sánchez Meca, se dio su nombre a la Subida de las Monjas ${ }^{27}$. La calle era de segundo orden, pero daba a la plaza del Ayuntamiento, ahora de García Hernández. Durante el segundo bienio republicano, el de la derecha en el poder, con el Ayuntamiento regido por una gestora conservadora desde octubre de 1934, no se produjo ningún cambio en el callejero. Tras el triunfo del Frente Popular en las elecciones del 16 de febrero de 1936, fue restablecido el Ayuntamiento del 14 de abril de 1931. En la sesión municipal del 13 de marzo de 1936, fue una petición vecinal la que impuso el cambio del nombre de una calle. De este modo la de Don Roque pasó a denominarse de Luis Sirval ${ }^{28}$. Se trató de la modificación de mayor calado político. El periodista Luis de Sirval -seudónimo de Luis Higón y Rosell- había sido asesinado el 27 de octubre de 1934 por los legionarios en la represión de la Revolución de Asturias. La demora en la solicitud obedecía a la etapa reaccionaria en que ocurrió. La vía era de segundo orden.

\section{GUÍA DE ITINERARIOS HISTÓRICOS}

Nodos e hitos constituyen los elementos vertebradores de la memoria urbana. Las calles y edificios emblemáticos fueron escenarios de hechos históricos que han sido olvidados o sepultados de forma interesada. A partir de estas consideraciones hemos establecido tres rutas: política, cultural y carcelaria, recuperando los lugares de la memoria republicana.

\section{RUTA POLÍTICA}

\section{La ciudad republicana}

Las calles ocupan un lugar destacado en la emergencia política. Lugares donde se respaldó el cambio político en la tarde del 14 de abril de 1931. El punto de arranque fue la Casa de los Republicanos en el $46-1^{\circ}$ de la Calle Mayor, donde se acababa de izar la bandera que los identificaba: "Seguidamente se organizó una manifestación que presidida por los componentes del Comité Republicano-Socialista se dirigió al Ayuntamiento de Cartagena, tremolando la bandera tricolor”. La multitudinaria exhibición de fervor y entusiasmo recorrió toda la calle Mayor, desembocando en la Plaza del Ayuntamiento: "La amplia plaza de Santa Catalina quedó prontamente invadida por la multitud que no cesaba de aplaudir y vitorear" ${ }^{29}$. La proclamación de la República se llevó a cabo desde el balcón del Palacio Consistorial allí emplazado.

La tensión acumulada se diluyó en festividad ${ }^{30}$. La celebración continuó en la jornada siguiente -15 de abril-, contemporizando las fuerzas militares: "Desde bien temprano se notó una extraordinaria animación en calles y plazas, contribuyendo a ello la declaración de fiesta nacional el día de hoy, por lo cual cesaron los trabajadores en el Arsenal y en la Constructora [Naval], cerrándose las escuelas nacionales y el Comercio. Manifestaciones de entusiasmo se originaron a diversas horas, recorriendo la banda de Infantería de Marina la población entonando la Marsellesa. / A las ocho por disposición del Gobierno provisional se izó la bandera republicana en el Palacio de Capitanía General y demás edificios oficiales y en los buques de guerra" ${ }^{31}$.

La Calle Mayor fue el centro de la celebración: “... estuvo animadísima a todas horas; los cafés llenos de gente y el himno de Riego y la Marsellesa sonando de cuando en cuando, entre la gente puesta en pie y descubierta"32. Fue paseado por la ciudad un cuadro alegórico de la República, finalmente instalado en el salón de sesiones del Consistorio. En aquel relevo de símbolos, alguien picó la corona real del escudo de la ciudad de Murcia de la Casa Llagostera, en la calle Mayor, respetando el emblema de Cartagena.

\footnotetext{
La Tierra (Cartagena), 4 de noviembre de 1933 y Cartagena Nueva (Cartagena), 4 de noviembre de 1933.

Cartagena Nueva, 14 de marzo de 1936 y El Noticiero (Cartagena), 14 de marzo de 1936.

El Eco de Cartagena, 15 de abril de 1931.

Ibidem.

Ibídem.

La Tierra (Cartagena), 16 de abril de 1931
} 


\section{El Arsenal}

El Arsenal Militar de Cartagena, construido entre 1732 y 1782 bajo la dirección primero del ingeniero Sebastián Feringán y luego de Mateo Vadopich, constituyendo un conjunto ilustrado de interés urbanístico, con amplias plazas, jardines, talleres y diques. En 1865 el ingeniero Tomás Tallaríe amplió su puerta neoclásica, adosándole una torre reloj de corte clasicista (Pérez Rojas, 1986: 322-323).

El edificio fue escenario privilegiado del final de la Monarquía. El rey obligado a abandonar España salió de Madrid en la noche del 14 de abril para dirigirse al exilio por el puerto Cartagena. Él mismo condujo uno de sus automóviles de lujo, un Duesemberg Convertible Town Car, carrozado en 1930 por Hibbard \& Darrin. Llegó a su destino a las cuatro de la madrugada del día siguiente, atravesando las puertas de acceso al Arsenal. Fue recibido por las autoridades militares, encabezadas por el almirante Magaz, capitán general del Departamento; el vicealmirante Ángel Cervera, comandante general del Arsenal; el general Zuvillaga, gobernador militar, y el general Murcia de Infantería de Marina. A las cinco, una lancha lo transbordó al crucero Príncipe Alfonso, que había de llevarlo a Marsella y que regresaría con el nombre de Libertad. El momento de la despedida delata el posicionamiento de aquellos mandos ${ }^{33}$.

La ausencia de incidentes en aquella hora decisiva, fue valorada por Alcalá Zamora: “... ha dado una prueba de un gran civismo, sabiendo terminar la revolución triunfante sin un choque, sin una gota de sangre, facilitando la escapada de la Monarquía; ya que la salida de Madrid era relativamente fácil, no así la salida de España. Cartagena, con todo civismo, puso al enemigo que huía puente de plata" ${ }^{34}$.

El Arsenal fue un lugar de relevancia política durante la etapa republicana. En agosto de 1934 se descubrió un complot anarquista. También la conspiración contra la República tuvo aquí uno de sus focos principales, convirtiéndose en el centro rebelde en el movimiento de julio de 1936. La sublevación acabó fracasando por la desconexión e irresolución de los golpistas y por la actitud enérgica del gobernador militar -Toribio Martínez Cabrera-, de algunos oficiales y, sobre todo, de los auxiliares, marinería y buena parte de los trabajadores y de la población civil (Martínez Leal, 1993).

\section{Ayuntamiento}

El Palacio Consistorial es una de las joyas de la arquitectura cartagenera. Está considerado como uno de los máximos exponentes del eclecticismo murciano (Pérez Rojas, 1986: 300). Fue construido entre 1900 y 1907, bajo la dirección del arquitecto Tomás Rico Valarino. Construido con materiales nobles reforzando su monumentalidad, como símbolo de autoridad, hito y posición estratégica (Ferrándiz, 2015: 24-25).

Epicentro de la vida política local, su ocupación representó el inicio de una nueva etapa histórica. El 14 de abril de 1931, a las seis de la tarde, siguiendo órdenes de Madrid, se proclamó la II República desde su balcón principal. Hicieron uso de la palabra los representantes de la Conjunción Republicano-Socialista, la coalición que había triundado en las elecciones del 12 de abril: Amancio Muñoz de Zafra, Antonio Ros, Severino Bonmatí, Francisco Pérez Lurbe, Ramón Navarro Vives y Ginés de Arlés García. Un grupo de exaltados irrumpió en las dependencias municipales destrozando a su paso los retratos del rey, respetando en cambio los de la reina ${ }^{35}$.

El alcalde saliente -Julio Mínguez- se negó a hacer entrega del poder local al no tener instrucciones del gobernador civil. A las doce la noche -sin esperar más- el Comité Republicano-Socialista se hizo cargo de la Corporación municipal. La gestora estaba integrada por los tres concejales más votados: Severino Bonmatí Vicedo (Partido Republicano Radical Socialista), Luis Romero Ruiz (Alianza Republicana) y Amancio Muñoz de Zafra (PSOE) (Martínez Leal, 1993: 90-93).

\footnotetext{
33 El Eco de Cartagena, 15 de abril de 1931.

34 Cartagena Nueva, 31 de marzo de 1932.

35 El Eco de Cartagena, 15 de abril de 1931.
} 


\section{Círculos políticos y sindicales}

Las calles -como hemos podido comprobar- cambiaron de nombre, convirtiéndose en referencia de la memoria que se quiere acuñar. En el itinerario propuesto se pueden recorrer siguiendo los planos del anexo documental, recordando el nombre que tuvieron y señalando sus nodos e hitos más relevantes.

En las calles de primer y segundo orden se encontraban las sociedades recreativas, deportivas y los círculos políticos de las clases medias y altas. En la calle Mayor: el Casino, el Club Victoria, la Peña X, la Unión Mercantil e Industrial, la Peña California, Cartagena F.C., Acción Democrática y el Círculo Federal. En la de Pi y Margall, el Partido Radical Autónomo; en la Plaza del Risueño Acción Republicana y en la de Prefumo, el Ateneo Mercantil. En el mismo muelle el Club de Regatas.

En algunas de segundo y en las de tercer orden se dan cita las sociedades obreras. La Casa del Pueblo amplía sus locales de la calle de Sambazart $\left(14-1^{\circ}\right)$ con el número 11 de la del Escorial. El Ateneo Sindicalista Libertario de la CNT y el Ateneo de Divulgación Social de la FAI pasan de San Antonio el Pobre junto con algunos sindicatos a la calle Jara, ahora de Salvador Seguí, mientras otras formaciones de la misma organización se reparten entre las calles Villamartín y Muralla del Mar.

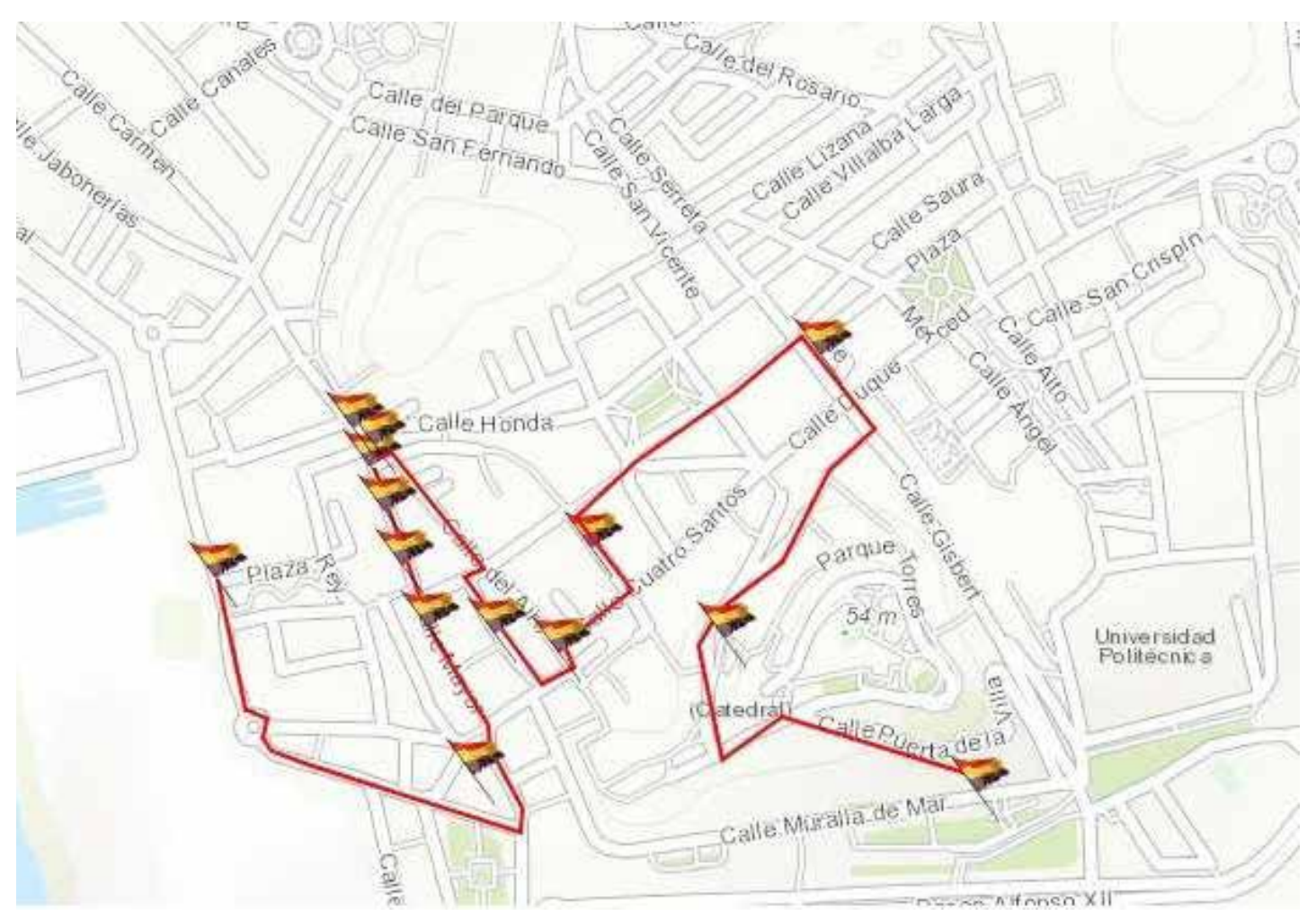

FIGURA 1. Ruta política

Fuente: Elaboración propia.

\section{RUTA CULTURAL}

\section{Escuelas}

La República apostó por la enseñanza. Una enseñanza laica que, con la Ley de Confesiones y Congregaciones Religiosas de 2 de junio de 1933, puso fin al monopolio de la Iglesia. En Cartagena había que suplir la impartida por los colegios regidos por los Hermanos Maristas (Sagrada Familia), los Padres Paules (Catedral Vieja) y, sobre todo, por las Hijas de la Caridad de San Vicente de Paúl: Patronato del Sagrado Corazón de Jesús, Asilo de San Miguel, Casa del Niño, Casa de la Misericordia, Asilo de la Rambla y Sagrado Corazón (barrio de Los Dolores). Entre todos atendían a 3.703 alumnos, sobre un conjunto de 5.100 escolares.

Se abrieron 103 escuelas, que cubrieron las necesidades de barrios y diputaciones, contemplando con singular interéslaatencióna las niñas. Para el núcleourbano se construyeron 
dos grupos escolares de 20 grados (10 de niños y 10 de niñas) en la Plaza de España (Carlos III) y Paseo de la Libertad (Alfonso XIII, San Isidoro y Santa Florentina). Su situación era inmejorable: jardines, arbolado y parterres, con terreno suficiente para recreo de los niños y fachada de clases orientada a mediodía. El proyecto se aprobó el 5 de septiembre de 1933 y la obra se concluyó en vísperas de la Guerra Civil. Proyectadas por el arquitecto municipal Lorenzo Ros, fueron los edificios más importantes levantados en Cartagena durante la II República, inspirados en los principios del racionalismo (Pérez Rojas, 1986: 286). Llevaron los nombres de Manuel Azaña y Marcelino Domingo. Iniciada la contienda se convirtió en la sede del Instituto de Segunda Enseñanza, denominándose García Lorca (Egea, 2006).

\section{Ateneo (Puertas de Murcia)}

La cultura se erigió en atención preferente. El Ateneo, ubicado en la céntrica Plaza de Prefumo (San Sebastián) fue su eje principal. La fachada del edificio -derruido en los años 80- no tenía nada de particular. Una construcción de dos plantas con ventanas y balcones y cubierta plana con terraza. Lo más interesante estaba en su interior con decoraciones modernistas en algunas de sus salas (Pérez Rojas, 1986: 342-343).

Se había fundado a mediados del siglo XIX con el nombre de Círculo Ateneo, pasando a denominarse Ateneo Mercantil e Industrial. La renovación de su dirección llegó a los pocos días de proclamarse la II República: "El Ateneo, privado ya de su dirección upetista, recobrará pronto, perdidas o transformadas las reminiscencias oscarinas, su carácter democrático y sus simpatías de siempre" ${ }^{36}$. Se preveía un nuevo tiempo de convivencia política plural, de democracia y cultura: "El Ateneo de Cartagena está llamado a ser un hogar amable para la juventud, llena ahora de ansias generosas, y para todos los demócratas que aparte de la filiación personal estricta, tendrán allí un refugio de convivencia política y social”37.

Lo presidió Casimiro Bonmatí, médico reconocido, presidente del Partido Republicano Radical Socialista y de la Casa de los Republicanos, alistándose luego en Izquierda Republicana, el partido liderado por Azaña. En aquel centro alternó la intelectualidad cartagenera, entre otros Carmen Conde, Antonio Oliver, Juan Lanzón, Rodríguez Cánovas, Vicente Ros, Ginés de Arlés García y Antonio Puig Campillo (Franco, 2005: 123-138).

Fue lugar de celebración de numerosos actos culturales, conferencias y conciertos como el de Agapito Marazuela ${ }^{38}$. Por lo demás, allí se impartían clases de gramática, inglés, francés, caligrafía, contabilidad, taquigrafía, mecanografía y primeras letras, llegando a contar hasta con 500 alumnos (Casal, 1933: 62). También abrió sus salones a las demandas de la población, convirtiéndose en el principal adalid en favor de la realización de los proyectos para dotar de aguas potables y de regadío a Cartagena y sus campos ${ }^{39}$.

\section{Universidad Popular}

Durante la II República prendió una nueva misión del saber, cuya mejor expresión fue la Universidad Popular. Su creación se forjó el 15 de julio de 1931 con la mediación de Ginés de Arlés García y Antonio Oliver, en una reunión a la que concurrieron representantes de la intelectualidad, de las organizaciones obreras, de los partidos republicanos, del Ayuntamiento y de la Prensa.

Se instaló en la calle Jara, pronto de Salvador Seguí. Su apertura oficial tuvo lugar el 12 de diciembre de 1931. La lección inaugural se impartió en el Ateneo. Corrió a cargo del diputado Fernando Valera, primer secretario de la comisión encargada de redactar la Constitución de 1931, que disertó sobre la Libertad de Conciencia ${ }^{40}$.

Su impacto social fue indudable. En julio de 1933 contaba con 563 afiliados, de los que 130 correspondían al primer curso y 433 al segundo. Se establecieron dos tipos de enseñanza. De un lado, las clases diarias de idiomas y Derecho, a las que acudían las clases trabajadoras.

\footnotetext{
36 La Tierra (Cartagena), 28 de abril de 1931. La dirección upetista y las reminiscencias oscarinas hacían referencia a Óscar Nevado, presidente del Ateneo durante la dictadura de Primo de Rivera y dirigente de la Unión Patriótica, la UP, de donde derivaba upetista, el partido del dictador

37 La Tierra (Cartagena), 28 de abril de 1931.

38 Cartagena Nueva, 27 de octubre de 1931.

39 Vid. El Porvenir (Cartagena), 9 de julio de 1931 y El Eco de Cartagena, 16 de noviembre de 1931.

40 La Tierra (Cartagena), 13 de diciembre de 1931.
} 
A ello se sumaban cursillos y conferencias impartidas por conocidos representantes de la cultura local: Antonio Puig Campillo, Lorenzo Ros Costa, Casimiro Bonmatí, Antonio Oliver y José Rodríguez Cánovas. A las clases para adultos se unían exposiciones y excursiones.

La otra vertiente de la docencia era la extensión universitaria, que se impartía en los salones del Ateneo. Para ello contaba con catedráticos de la Universidad de Murcia y destacados representantes de la intelectualidad nacional: Mariano Ruiz-Funes, José Loustau, Fernando Valera, Ramón Sijé, Miguel Hernández, Luis Calandre, Victoria Kent, María Cegarra o Margarita Nelken, por citar algunos (Franco, 2005: 138-146).

La Universidad Popular actuó además como medio de enlace con el Patronato de Misiones Pedagógicas de la República. Dispuso por este medio de una biblioteca circulante de 350 volúmenes, contabilizando 2.600 lecturas en 1933 (Casal, 1933: 95-99).

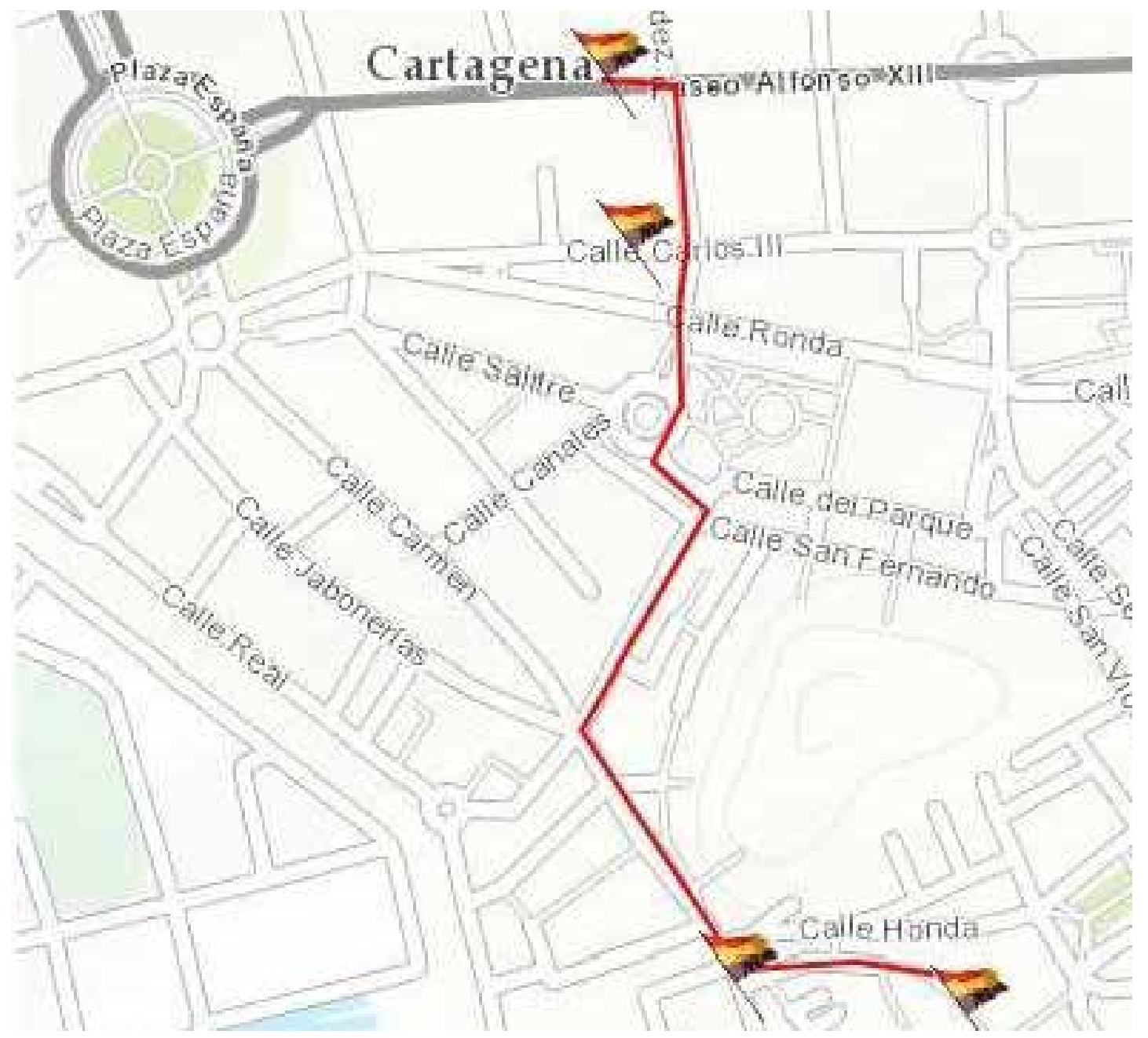

FIGURA 2. Ruta cultural

Fuente: Elaboración propia.

\section{RUTA CARCELARIA}

En 1930, según el Reglamento de los Servicios de Prisiones, las cárceles dependientes del Ministerio de Justicia -entonces de Gracia y Justicia- eran de tres clases: centrales, provinciales y de partido. Cartagena contaba con la correspondiente cárcel de partido, emplazada en el barrio de San Antonio Abad, y con una prisión central -el Penal-, que formaba parte de la red nacional junto con las del Dueso, San Miguel de los Reyes (Valencia), Burgos, Santa María del Puerto, Figueras y los reformatorios de Ocaña y Alicante ${ }^{41}$. A ello había que sumar las dependencias militares instaladas en el Arsenal -la denominada Grillera- y el Castillo de San Julián.

41 Gaceta de Madrid, 21 de noviembre de 1930, pp. 1.030-1.032. 


\section{El Presidio}

El Presidio fue construido entre 1776 y 1785 con la finalidad de confinar a los forzados de galeras. Adosado al Arsenal, dependía de la Marina, que lo reclamará con frecuencia. Era conocido como la Casa Negra. A pesar de su vetustez no fue acondicionado hasta 1911 (Chacón Bulnes, 2012).

La denominada -sin duda con razón- Prisión de Penas Aflictivas, estaba considerada como uno de los penales más ingobernables y de condiciones higiénicas menos apropiadas: “... la penitenciaría de Cartagena es un foco permanente de infección que perjudica a la salud pública y causa, la más abonada, para el desarrollo y propagación de las epidemias". Era sinónimo de conmoción y trastorno: "Las continuas huelgas, plantes y riñas tenían en diaria alarma a toda la población que llegó a pedir el traslado de dicho Penal, por amenazar la tranquilidad pública al ser tan frecuentes las alteraciones del orden"42. Su situación no comenzaría a mejorar hasta 1911, bajo la dirección de Ricardo Mur Grande, de apellidos más que apropiados. Se hizo entonces la fachada de un clasicismo modernista que todavía se conserva (Pérez Rojas, 1986: 295).

El Penal era toda una referencia como lugar de castigo. En 1917 había albergado al comité de la huelga de agosto de aquel año. Se trataba de destacados dirigentes del PSOE y la UGT, nombres ilustres de la política, del mundo sindical y de la Universidad: Daniel Anguiano, Largo Caballero, Julián Besteiro y Andrés Saborit.

Era algo consustancial a la ciudad. Su visibilidad resultaba manifiesta, particularmente cuando las cuerdas de presos recorrían sus calles cardinales: "Casi a diario llegan a la estación del ferrocarril presos confinados a esta prisión aflictiva, conducidos por la guardia civil. Estos pobres penados tienen que ir de pie, esposados, y atravesar las principales vías de la población, llamando la atención de cuantos transitan por ellas, siendo esto motivo de un espectáculo poco edificante, pues sirve de compasión a las gentes generosas y de mofa a los incultos"33.

Durante la II República la institución estuvo dirigida por Diego Robles Martínez, siendo su administrador Pedro Bernal y su médico, Felipe Reverte (Casal, 1933: 89). Nada parece indicar que se deteriorase su habitabilidad. En mayo de 1931, la directora general de Prisiones -en revista oficial- se llevó las mejores impresiones: "La señorita Kent visitó con todo detenimiento la Prisión Aflictiva, haciendo grandes elogios de los empleados de dicha casa y de las distintas dependencias en cuanto a condiciones higiénicas y de comodidad" 4 .

El 16 de abril de 1931 -a los dos días de proclamarse la República- cumpliendo órdenes del Gobierno Provisional, comenzó a aplicarse el decreto de amnistía ${ }^{45}$. Al día siguiente hubo un atisbo de motín al considerar los comunes que el indulto concedido era muy limitado. Entre los primeros libertados figuraba Domingo Marsach, que se encontraba cumpliendo condena por atentado a Primo de Rivera en Barcelona ${ }^{46}$.

En enero de 1931 su población ascendía a 560 reclusos. La amnistía decretada por la República redujo la cifra a 213, descendiendo a 170 en 1932 y aumentando ligeramente en 1933: $215^{47}$. Nada que ver con las cifras de 1934, cuando con la represión del movimiento revolucionario de octubre por un gobierno de derechas superó los 600 penados, de los cuales 400 eran socialistas y sindicalistas (Egea, 2011: 225 y 229).

Uno de ellos fue Ramón González Peña, ex diputado socialista y principal dirigente del levantamiento asturiano. Estuvo recluido hasta el 23 de junio, cuando fue trasladado a la cárcel de Chinchilla. La marcha de González Peña se hizo coincidir con la llegada de algunos de los ex consejeros de la Generalidad catalana condenados a 30 años de cárcel por el Tribunal de Garantías por su participación en la declaración de la República Catalana: Martí Barrera, Pere Mestres, Ventura Gassol y Martí Esteve.

\footnotetext{
El Porvenir (Cartagena), 21 de octubre de 1918.

La Tierra (Cartagena), 14 de septiembre de 1933.

La Tierra (Cartagena), 12 de mayo de 1931.

Cartagena Nueva, 18 de abril de 1931.

La Tierra (Cartagena), 18 de abril de 1931.

INE: Anuario Estadístico de España. Años 1931, 1932-1933 y 1934.
} 
El último acontecimiento vivido durante la II República tuvo lugar el 17 de febrero de 1936 cuando estalló otro motín. En la revuelta alentaba el resentimiento de los comunes contra los internos políticos, liberados tras el triunfo electoral del Frente Popular. Lo ocurrido trató de ser explotado por las expectativas políticas en juego. La derecha -siguiendo su estrategia de la tensión, ya bien perfilada-, estuvo interesada en magnificar el altercado (Egea, 2011).

El Penal fue clausurado en 1946, reconvirtiéndose -con las obras oportunas- en Cuartel de Instrucción de Marinería. Apenas contaba entonces con 40 presos, la mayoría comunes ${ }^{48}$. En la actualidad alberga la Facultad de Ciencias de la Empresa de la Universidad Politécnica de Cartagena.

\section{La cárcel de San Antón}

La cárcel del partido ofrecía una importancia mucho menor. A finales del siglo XIX podía alojar, por término medio, a unos 45 sujetos. Era un viejo caserón emplazado en el arrabal de San Antonio Abad, junto a la iglesia, que había sido almacén de granos. Su habitabilidad no podía ser más deficiente: “... patios pequeños, cuadras húmedas y mal ventiladas, con retretes sin condiciones...”. Fue considerada, con las de Almería y Alicante, “... de las peores que existen”. ${ }^{9}$

Su estado de abandono era absoluto: "Aquellos reclusos carecen de todo. Sólo un rancho pobre y malo, lo indispensable para no morirse en cuatro días, es lo que permite darles la exigua consignación del Estado, que pasa para todas las atenciones una peseta por cabeza. Como además las estrecheces del local no les permite montar talleres, donde con su trabajo puedan ayudarse a las necesidades de la vida, se da el caso de reclusos que entran sin más que lo puesto, y eso roto y sucio, y no se les puede cambiar de ropa, porque la cárcel no tiene ropero ni medios de establecerlo. Y si eso pasa con la ropa, calcúlese como estarán las camas y el servicio de medicinas y enfermería" ${ }^{50}$. Allí -contra toda razón- eran recluidos los niños delincuentes, aunque no faltasen voces que pidieran la habilitación de un reformatorio ${ }^{51}$.

Su situación no había dejado de empeorar. Cuando el 11 de mayo de 1931 lo visitó Victoria Kent, propuso su clausura inmediata ${ }^{52}$. El proyecto de un nuevo centro se aprobó el 12 de octubre de 1933, dotándose con un presupuesto de 859.456,24 pesetas ${ }^{53}$. Se debió a Vicente Agustí Elguero, arquitecto de la Dirección General de Prisiones, que también realizó la Cárcel de Carabanchel de Madrid. Considerada como ejemplo de arquitectura carcelaria, posee una galería central, con una planta de cruz, desde cuyo centro se puede vigilar a todo el interior. Se inauguró el 31 de julio de 1936, ocupándose en septiembre con presos políticos, los implicados en la conspiración contra la República. Uno de ellos, José García Segado, el maestro de obras de su construcción.

\section{Castillo de San Julián}

Su construcción se inició en 1706 con una torre levantada por los ingleses en el contexto de la Guerra de Sucesión, ideada para la defensa del puerto de Cartagena, remodelándose entre 1861 y 1888, lo que explica la diversidad de estilos, dominando el neoclasicismo ecléctico (Gómez Vizcaíno, 1997: 94).

Fue escenario del levantamiento republicano de 1886, que le costó la vida al defensor de la fortaleza -General Fajardo- y al impulsor del asalto: Manuel Bartual Verdejo, al que se le dedicó una calle en 1931.

Durante la II se convirtió en lugar de confinamiento de los militares implicados en la revolución de octubre de 1934: el teniente coronel de Infantería Juan Ricart March -jefe de la Guardia de Asalto y Seguridad de Barcelona-, el comandante de Artillería Enrique Pérez Farrás -jefe de los Mozos de Escuadra- y los tenientes de la Guardia Civil Fernando Condés Romero -que intervino en los conatos de insurrección de Madrid- y Gabriel Torrens Llombart, que colaboró con el comité revolucionario de Asturias.

\footnotetext{
El Noticiero (Cartagena), 25 de enero de 1946.

El Porvenir (Cartagena), 5 de mayo de 1923.

Ídem.

El Noticiero (Cartagena), 4 de noviembre de 1943.

La Tierra (Cartagena), 12 de mayo de 1931.

Gaceta de Madrid, 14 de octubre de 1933, p. 387.
} 


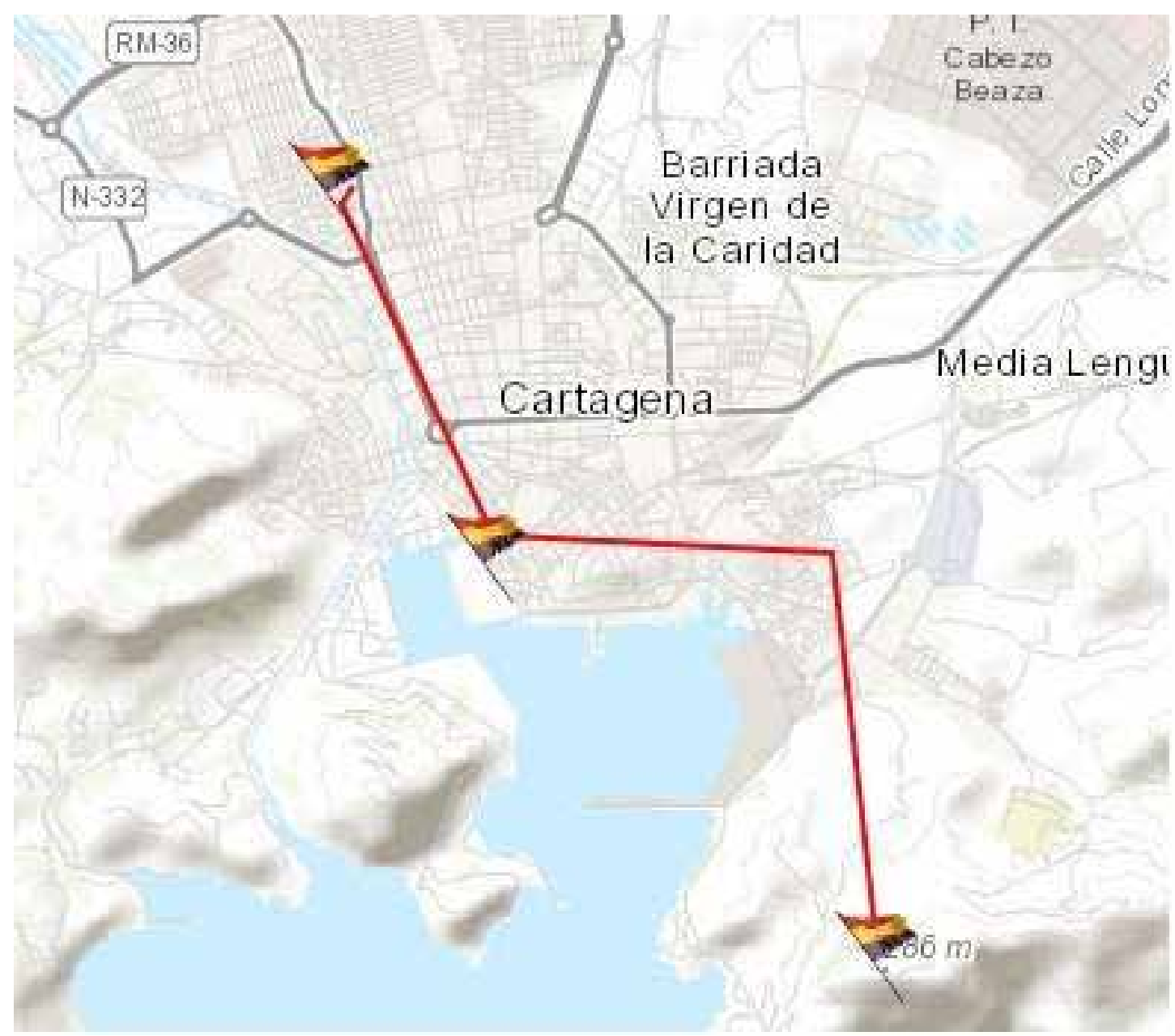

IMAGEN 3. Ruta carcelaria

\section{CONCLUSIONES}

Es posible recuperar la memoria histórica de Cartagena desde el punto de vista patrimonial. La bibliografía consultada permite contar con el marco histórico necesario para contextualizar el trazado urbano e histórico de la ciudad para disponer de una estructura donde articular la lectura de los símbolos que subyacen en las calles y en las piedras cargadas de pasado histórico.

El estudio de la memoria figura desde hace años como un nuevo campo historiográfico. Dentro de la disciplina los lugares de la memoria -leyendo a Pierre Nora- se convierten en elementos simbólicos del patrimonio memorial de cualquier comunidad.

Siguiendo a Kevin Lynch la ciudad se organiza en cinco elementos definidos por sus connotaciones sociales: sendas, bordes, barrios, nodos e hitos. Por su especial relevancia en el estudio abordado hemos dedicado una especial atención a las sendas, los nodos y los hitos.

Las calles de la ciudad de Cartagena se clasificaban desde el Ayuntamiento en órdenes según su categoría social. En las de primero y segundo se desarrolló el transcurrir de las clases altas y fueron escenario de celebraciones festivas y de todo tipo de manifestaciones, dado su mayor impacto mediático. Allí se emplazaban los centros de sociabilidad, las sedes políticas y sindicales y las entidades culturales, con los edificios más emblemáticos: Ayuntamiento, Arsenal, Universidad Popular, Penal...

Los rótulos de las calles ocupan un lugar relevante en la interpretación del pasado, cargados como están de simbolismo político. Vienen a ser el mapa mudo de una ciudad que hay que saber leer e interpretar. Durante la II República, especialmente durante el bienio social-azañista, fueron sustituidas las menciones religiosas, militares y monárquicas por denominaciones vinculadas a las nuevas coordenadas democráticas.

Con la información recopilada y los análisis realizados hemos articulado tres itinerarios histórico-patrimoniales: político, cultural y carcelario. Destacados lugares, en los 
que el contexto histórico, que permite explicar la relevancia del lugar, se completa con datos relativos al patrimonio arquitectónico.

La ruta política plantea un recorrido por la ciudad republicana, por las calles y lugares donde se respaldó el cambio político, empezando por la Casa de los Republicanos, en plena Calle Mayor. Son sus hitos el Ayuntamiento, desde cuyo balcón se proclamó la II República, el Arsenal, lugar elegido para la salida de España de Alfonso XIII y centro de conspiración de distinto signo. De no menor relieve son los círculos políticos y sindicales que emergieron aquí y allá por todo el casco de la población y las vías donde se asientan, muchas de ellas cambiadas de nombre.

La ruta cultural incluye las escuelas creadas por la República, la obra más importante de la etapa; el Ateneo, el centro de mayor difusión cultural de la ciudad y la Universidad Popular, firmemente conectada al proyecto educativo del Patronato de Misiones Pedagógicas de la República.

La ruta carcelaria permite conocer los lugares de represión de aquellos años, empezando por el viejo Presidio -la Prisión de Penas Aflictivas-, la Cárcel de San Antón, inaugurada el 31 de julio de 1936 con los primeros presos políticos implicados en la conspiración contra la República. Cierra este amplio recorrido el Castillo de San Julián, lugar de reclusión de los militares, en uno de los emplazamientos más bellos de la costa cartagenera. 


\section{BIBLIOGRAFÍA}

- Andrés Sarasa, J. L. (1981).Cartagena: estudio de geografía urbana. Murcia: Secretariado de Publicaciones de la Universidad de Murcia.

- Cabrera, M. A. (2010). La investigación histórica y el concepto de cultura política. En Culturas politicas: teoría e historia. Zaragoza: Institución Fernando el Católico, Zaragoza, 19-85.

- Casal Martínez, F. (1930) [1986]. Historia de las calles de Cartagena. Cartagena: Imp. Vda. de M. Carreño, 1930. $2^{\mathrm{a}}$ ed. Murcia, Academia Alfonso X el Sabio.

- Casal Martínez, F. (1933). Nuevo libro de la ciudad de Cartagena y su término municipal: comercio, industria, turismo (guía oficial). Cartagena: Imp. Vda de M. Carreño.

- Celdrán, J. A. (2008). Los espacios urbanos de la ciudad de Cartagena y sus procesos de reforma. Kesse, 42, 9-16.

- Chacón Bulnes, J. M. (2012). La Casa Negra: el Cuartel de Presidiarios y Esclavos de Cartagena. Cartagena: Universidad Politécnica de Cartagena.

- Cuesta Bustillo, J. (1998). Memoria e historia. Un estado de la cuestión. Ayer, 32, 203-246.

- Egea Bruno, P. M. (1996). Los siglos XIX y XX. En Manual de Historia de Cartagena. Cartagena: Ayuntamiento de Cartagena, Universidad de Murcia y Caja de Ahorros del Mediterráneo.

- Egea Bruno, P. M. (1999). Cartagena, imagen y memoria. Murcia: Arte Libro - Rafael Amorós.

- Egea Bruno, P. M. (2006). La enseñanza primaria en Cartagena durante la II República y la Guerra Civil (1931-1939). Cartagena: Áglaya.

- Egea Bruno, P.M. (2011). La amnistía de febrero de 1936 en Cartagena: Verdad y versión. Aproximación a las claves de un proceso de mistificación política. Espacio, Tiempo y Forma. Serie V, Historia Contemporánea, 23, 221-242.

- Erice, F. (2009). Guerras de la memoria y fantasmas del pasado. Usos y abusos de la memoria colectiva. Oviedo: Ed. Eikasia.

- Ferrándiz Araujo, V. M. (2015). El Palacio Consistorial de Cartagena, ejemplo de arquitectura institucional en la España del cambio de siglo XIX-XX. $P+C, 6,19-32$.

- Franco, F.J. (2002). Cartagena (1931-1936). Los años de la esperanza. Cartagena: Áglaya.

- Franco, F. J. (2005). República, guerra y exilio. Antonio Ros y la Generación del 27. Cartagena: Áglaya.

- Gómez Vizcaíno, A. (1997). Castillos y fortalezas de Cartagena. Cartagena: AFORCA.

- Henales Salamanca, J. (2017). El callejero histórico de Alicante: de la II República a nuestros dias. Lugares de historia y de memoria. Tesis doctoral dirigida por José Miguel Santacreu Soler: Universidad de Alicante.

- Lynch, K. (2015). La imagen de la ciudad. Barcelona: Gustavo Gili.

- Martínez Leal, J. (1993). República y Guerra Civil en Cartagena (1936-1939). Cartagena: Ayuntamiento de Cartagena-Universidad de Murcia.

- Nora, P. (2008). Pierre Nora en Les lieux de mémoire. Montevideo: Trilce.

- Pérez Rojas, J. (1986). Cartagena 1874-1936. (Transformación urbana y arquitectura). Murcia: Editora Regional de Murcia.

- Rodríguez Martín, J. A. (2013). Renovación de la ciudad, Cartagena 1875-1936. Guía de Arquitectura de Cartagena. Trabajo Fin de Máster de Patrimonio Arquitectónico: Universidad Politécnica de Cartagena.

- Ros Mcdonell, D. (2010). Impacto ambiental de las construcciones militares en Cartagena y su entorno. $P+C, 1,69-82$.

- Sánchez Costa, F. (2009). Los mapas de la memoria. Nombres de calles y políticas de la memoria en Barcelona y Madrid. En Hispania Nova, 9, http://hispanianova.rediris.es. (Consultado en 14/02/2018).

- Victoria Moreno, D. (1993). Las calles de Cartagena: expresión de la conciencia revolucionaria de un pueblo. El infortunado epílogo (1931-1942). Agua, 18, 18-19.

- Vinyals I Garrido, A. (2016). Being republican during the Second Spanish Republic. The articulation of a republican people and its limits. En La Segona República. Cultures i projectes politics. Congrés Internacional d'História. Barcelona: Universidad Autónoma de Barcelona.

- Wollentz, G. (2014). The Cultural Heritage as a Resource in Conflict Resolution. An Overview of the Field. Kalmar (Suecia): Kalmar Lans Museum. 